\title{
MnAs Nanocrystals Embedded in GaAs
}

\author{
A. Kwiatkowski ${ }^{a}$, J. Borysiuk $^{a, b}$, R. BożEK ${ }^{a}$, D. WASIK $^{a}$, \\ M. KAmińskA ${ }^{a}$, J. SAdowski ${ }^{c}$ AND A. TWARDOWski ${ }^{a}$ \\ ${ }^{a}$ Institute of Experimental Physics, Warsaw University \\ Hoża 69, 00-681 Warsaw, Poland \\ ${ }^{b}$ Institute of Electronic Materials Technology \\ Wólczyńska 133, 01-919 Warsaw, Poland \\ ${ }^{c}$ Institute of Physics, PAS, al. Lotników 32/46, 02-668 Warsaw, Poland
}

We report on cross-sectional transmission electron microscopy and magnetic force microscopy studies performed on self-organized MnAs nanoclusters embedded in GaAs. It was found that $10 \div 20 \mathrm{~nm}$ large MnAs ferromagnetic nanocrystals were formed during rapid thermal annealing of $\mathrm{Ga}_{1-x} \mathrm{Mn}_{x}$ As layers at $600^{\circ} \mathrm{C}$, leading to magnetic contrasts in magnetic force microscopy images.

PACS numbers: 61.46.+w, 61.82.Fk, 68.37.Rt, 68.37.Lp, 74.25.Ha

\section{Introduction}

In the last years a lot of effort has been devoted to a search for hybrid ferromagnet-semiconductor structures in aim of novel information storage and spin electronics applications. Nanocomposites with ferromagnetic above room temperature MnAs nanoclusters, embedded in GaAs matrix (MnAs/GaAs), are promising candidates for this type of material (e.g. [1-3]). Recently, it has been reported that thermal annealing of $\mathrm{Ga}_{1-x} \mathrm{Mn}_{x}$ As layers leads to formation of MnAs dots (e.g. $[4,5])$. In this work we performed systematic studies of a series of $\mathrm{Ga}_{1-x} \mathrm{Mn}_{x} \mathrm{As}$ layers, annealed at different temperatures, in order to find correlations between structural and magnetic properties of resulting composite (Mn,Ga)As material.

\section{Samples and experiments}

All the samples were manufactured by molecular beam epitaxy (MBE) technique. Self-organized MnAs nanoclusters were formed during thermal annealing of $\mathrm{Ga}_{1-x} \mathrm{Mn}_{x}$ As layers $(L=50 \mathrm{~nm}$ or about $L=1 \mu \mathrm{m}$ thick) grown on $\operatorname{GaAs}(100)$ substrates at about $230^{\circ} \mathrm{C}$ and then covered by amorphous As ( $1 \mu \mathrm{m}$ thick) or low temperature LT-GaAs (50 nm thick). Mn content $x$ was $2 \%, 5 \%$, or $6 \%$. The 
annealing was performed in As flux at different temperatures, $T=500^{\circ} \mathrm{C}, 550^{\circ} \mathrm{C}$, or $600^{\circ} \mathrm{C}$. As-grown $\mathrm{Ga}_{1-x} \mathrm{Mn}_{x} \mathrm{As} / \mathrm{GaAs}$ heterostructure, without any heat treatment, was used as a reference sample. Details of the growth procedure are given in $[4]$.

Transmission electron microscopy (TEM) was used to study structural properties of the (Ga,Mn)As layers. Cross-sectional TEM specimens were prepared by a standard method of mechanical pre-thinning followed by Ar ion milling. Conventional and high-resolution (HRTEM) observations were performed on a JEOL JEM 3010 microscope operating at $300 \mathrm{kV}$.

Surface topography and microscopic magnetic structure were studied at room temperature using Digital Instruments MultiMode scanning probe microscope operating in a tapping-lift mode. Magnetic tips, MESP-HM, having dipole moment higher than $3 \times 10^{-13}$ emu were used for image acquisition. The tips were magnetized vertically, along the needle, with a permanent magnet. The shift of cantilever resonant frequency is approximately proportional to the gradient in the vertical direction of the vertical component of the magnetic force. The magnetic images were recorded using a standard procedure. First, the topography of the surface was determined (tapping mode), then the needle followed the same path keeping constant height above the surface (lift mode) and recording frequency changes of cantilever vibration induced by the gradient of magnetic field.

\section{Results and discussion}

The results of cross-sectional TEM measurements are presented in Fig. 1. The TEM observations indicated that annealing of $\mathrm{Ga}_{1-x} \mathrm{Mn}_{x}$ As layers led to local changes of their chemical composition, while the as-grown layers were chemically homogeneous. Annealing at $500^{\circ} \mathrm{C}$ resulted in a considerable diffusion of $\mathrm{Mn}$ and, what was more - its segregation. Mn-rich zinc blende $\mathrm{Mn}(\mathrm{Ga})$ As clusters coherent with GaAs matrix were observed. These clusters had typical sizes of $1.5 \div 2 \mathrm{~nm}$ for a layer with $2 \%$ of $\mathrm{Mn}$ and they did not show structural defects at their boundaries. Therefore, obviously the segregation process caused the non-uniform strain in such layer. For higher initial Mn contents, i.e. for $5 \%$ or $6 \%$ of Mn, TEM measurement showed $3 \mathrm{~nm}$ clusters with pronounced structural disorder. Annealing at higher temperature of $550^{\circ} \mathrm{C}$ led to creation of larger clusters, more distorted, and in some cases to formation of regular MnAs hexagonal precipitates. Most likely, some small clusters observed for samples annealed at $500^{\circ} \mathrm{C}$ were dissolved during $550^{\circ} \mathrm{C}$ annealing, but others grew in size and created larger disordered clusters. The size of disordered clusters depended on the initial Mn content. For $2 \%$ of Mn the cluster diameter was about $3 \mathrm{~nm}$, while for $5 \div 6 \%$ of $\mathrm{Mn}$ content it reached about $5 \mathrm{~nm}$. For the highest reported annealing temperature of $600^{\circ} \mathrm{C}$ spherical nanocrystals were observed. They had well-developed MnAs hexagonal structure (see Fig. 1). Their diameter was about $10 \mathrm{~nm}$ for $2 \% \mathrm{Mn}$ initial content and up to $20 \mathrm{~nm}$ for $5 \div 6 \%$ of Mn. Most of MnAs clusters were coherent with GaAs matrix, but some of them revealed weak structural disorder at their boundaries. 

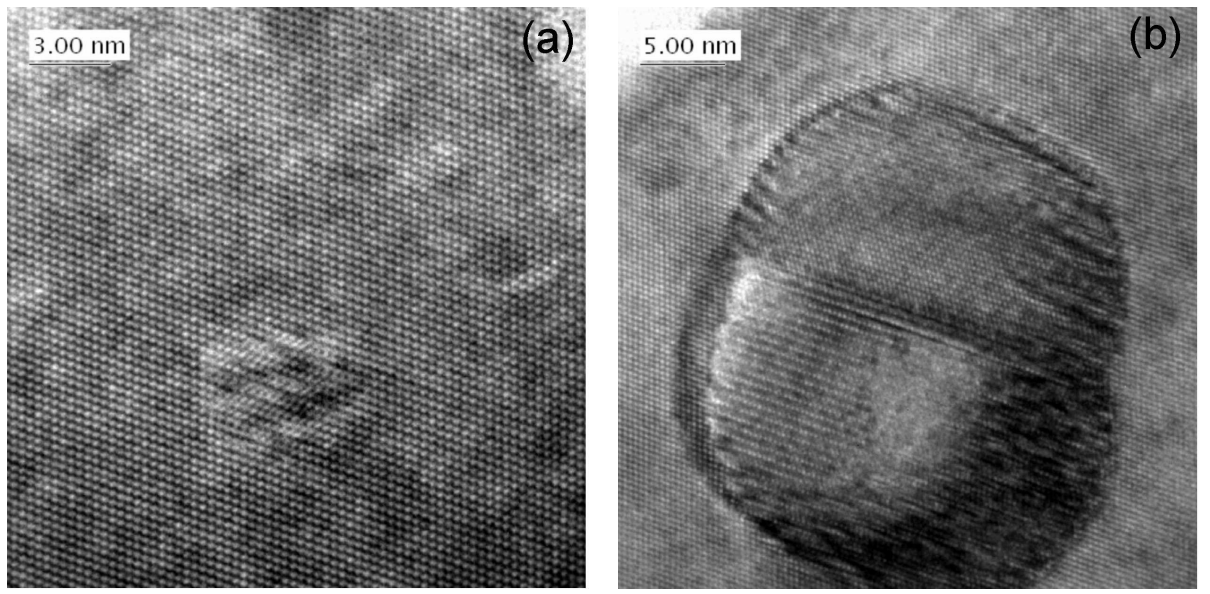

Fig. 1. High resolution X-ray TEM (XTEM) images of two types of nano-objects observed in the studied samples. (a) Mn-rich zinc-blende (Ga,Mn)As cluster coherent with GaAs matrix observed in the samples annealed at $500^{\circ} \mathrm{C}$, (b) MnAs nanocrystal of NiAs (hexagonal) structure observed in the samples annealed at $600^{\circ} \mathrm{C}$.

Similar set of annealed layers was also studied by atomic force microscopy/magnetic force microscopy. The respective images are presented in Fig. 2 for two representative samples annealed at $500^{\circ} \mathrm{C}$ and $600^{\circ} \mathrm{C}$.
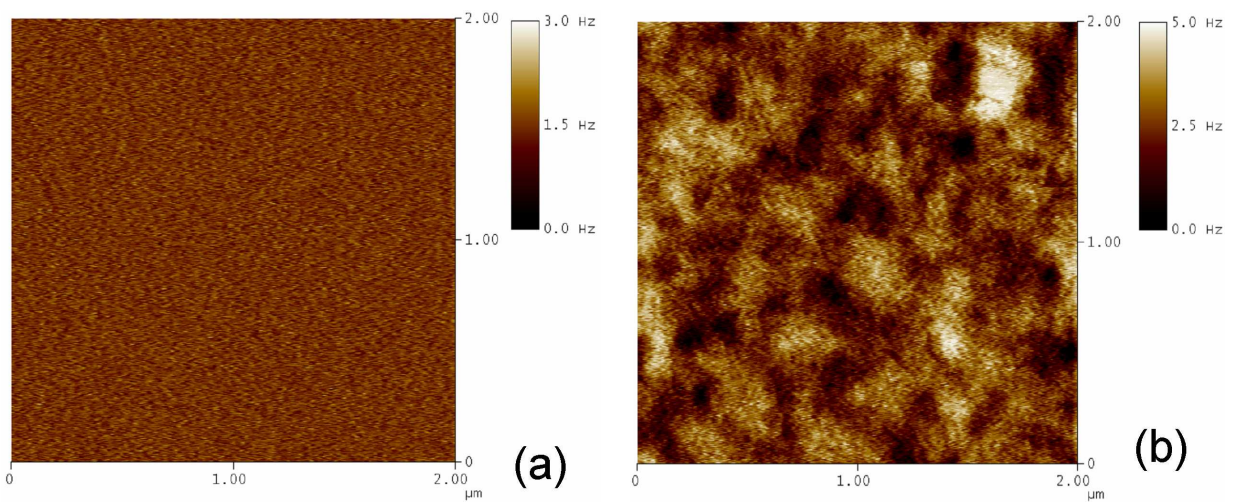

Fig. 2. Magnetic force microscopy images of the samples annealed at (a) $500^{\circ} \mathrm{C}$, (b) $600^{\circ} \mathrm{C}$.

We found that all the samples annealed at $600^{\circ} \mathrm{C}$ revealed some magnetic contrast, while uniform images with no magnetic contrast were recorded for as-grown and annealed at $550^{\circ} \mathrm{C}$ or $500^{\circ} \mathrm{C}$ layers. These results correlated well with TEM results and unambiguously proved that $10 \div 20 \mathrm{~nm}$ large MnAs clusters were ferromagnetic at room temperature. The smaller dots were not observed in magnetic force microscopy images. The reason was that either they were not fer- 
romagnetic at all, or their Curie temperature was below room temperature, or the contrasts were too small for detecting by magnetic tip used in our experiments.

Up to our knowledge, this paper is the first report on magnetic contrasts observed in magnetic force microscopy images, originating from MnAs ferromagnetic nanocrystals located about $10 \div 20 \mathrm{~nm}$ under surface of the measured samples. We proved that magnetic force microscopy is a valuable technique to monitor magnetic objects of nanosize in composite materials. The presented work is a first step to obtain ferromagnetic nanoprecipitates embedded in semiconductor matrix, of desired size and density.

\section{Acknowledgments}

The use of TEM facilities at the Faculty of Materials Science and Engineering of Warsaw University of Technology is kindly appreciated.

This work was partially supported by the State Committee for Scientific Research — grant: KBN 7T08A03528.

\section{References}

[1] M. Moreno, A. Trampert, B. Jenichen, L. Däweritz, K.H. Ploog, J. Appl. Phys. 92, 4672 (2002).

[2] M. Moreno, B. Jenichen, V. Kagner, W. Braun, A. Trampert, L. Däweritz, K.H. Ploog, Phys. Rev. B 67, 235206 (2003).

[3] T. Plake, T. Hesjedal, J. Mohanty, M. Kästner, L. Däweritz, K.H. Ploog, Appl. Phys. Lett. 82, 2308 (2003).

[4] M. Adell, L. Ilver, J. Kanski, V. Stanciu, P. Svedlindh, J. Sadowski, J.Z. Domagala, F. Terki, C. Hernandez, S. Charar, Appl. Phys. Lett. 86, 112501 (2005).

[5] J. Sadowski, E. Janik, E. Łusakowska, J.Z. Domagala, S. Kret, P. Dłuzewski, M. Adell, J. Kanski, L. Ilver, R. Brucas, M. Hanson, Appl. Phys. Lett. 87, 263114 (2005). 